This article was downloaded by:[Swets Content Distribution]

On: 26 July 2007

Access Details: [subscription number 768307933]

Publisher: Psychology Press

Informa Ltd Registered in England and Wales Registered Number: 1072954

Registered office: Mortimer House, 37-41 Mortimer Street, London W1T 3JH, UK

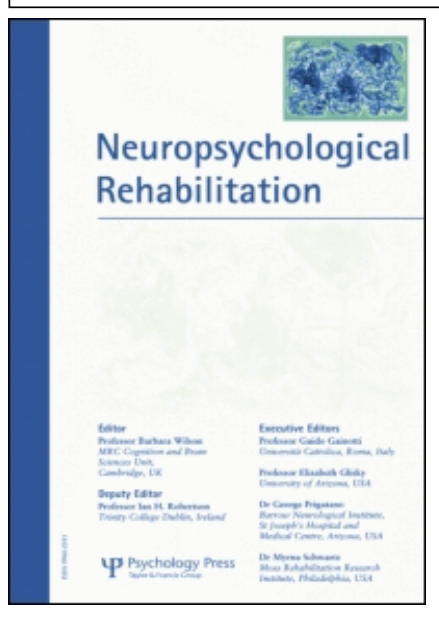

\title{
Neuropsychological Rehabilitation
} An International Journal

Publication details, including instructions for authors and subscription information: http://www.informaworld.com/smpp/title content=t713684225

The application of errorless learning to aphasic disorders: A review of theory and practice Joanne K. Fillingham ${ }^{\text {a; }}$ Catherine Hodgson ${ }^{\text {a }}$; Karen Sage ${ }^{\text {a; }}$ Matthew A. Lambon Ralph $^{\text {a }}$

a University of Manchester, UK.

Online Publication Date: 01 July 2003

To cite this Article: Fillingham, Joanne K., Hodgson, Catherine, Sage, Karen and Ralph, Matthew A. Lambon (2003) 'The application of errorless learning to aphasic disorders: A review of theory and practice', Neuropsychological Rehabilitation, 13:3, $337-363$

To link to this article: DOI: $10.1080 / 09602010343000020$

URL: http://dx.doi.org/10.1080/09602010343000020

\section{PLEASE SCROLL DOWN FOR ARTICLE}

Full terms and conditions of use: http://www.informaworld.com/terms-and-conditions-of-access.pdf

This article maybe used for research, teaching and private study purposes. Any substantial or systematic reproduction, re-distribution, re-selling, loan or sub-licensing, systematic supply or distribution in any form to anyone is expressly forbidden.

The publisher does not give any warranty express or implied or make any representation that the contents will be complete or accurate or up to date. The accuracy of any instructions, formulae and drug doses should be independently verified with primary sources. The publisher shall not be liable for any loss, actions, claims, proceedings, demand or costs or damages whatsoever or howsoever caused arising directly or indirectly in connection with or arising out of the use of this material.

(C) Taylor and Francis 2007 


\title{
The application of errorless learning to aphasic disorders: A review of theory and practice
}

\author{
Joanne K. Fillingham, Catherine Hodgson, \\ Karen Sage, and Matthew A. Lambon Ralph \\ University of Manchester, $U K$
}

\begin{abstract}
Recently, there has been great interest in errorless learning as a new intervention technique. This may be because there are data from both basic neuroscience and clinical application that suggest it is superior to more traditional trial and error methods. In the contemporary literature the most prominent investigations of errorless learning are those designed for rehabilitation of memory impairments. These studies include numerous demonstrations of effective amelioration of word finding difficulties using errorless intervention. The aphasic literature contains no examples of purely errorless learning methods, however, some studies have successfully treated anomia using error reducing techniques. This suggests that errorless learning could be advantageous for treating aphasic disorders, in particular anomia. To explore this idea, we discuss previous research and review two current theories of errorless learning. We provide our own framework for thinking about errorless and errorful learning. In addition, a review of the anomia treatment literature (from 1985 to the present) is presented and conclusions drawn on the methods of treating the word finding difficulties of patients with aphasia.
\end{abstract}

\section{INTRODUCTION}

Neural plasticity, that is the ability of the brain to change its function, is a current and fundamental issue in neuroscience (e.g., Buonomano \& Merzenich, 1998; Tallal, Merzenich, Miller, \& Jenkins, 1998). Work at this level of basic science has shown that the mature brain is, in principle, capable of "rewiring" itself so that new functions can be learnt by brain areas, which previously performed other processes (Buonomano \& Merzenich, 1988). This basic

Correspondence should be addressed to Joanne Fillingham or Prof. Matthew Lambon Ralph, Department of Psychology, The University of Manchester, Oxford Road, Manchester, M13 9PL, UK. Email: joanne.k.fillingham@man.ac.uk or matt.lambon-ralph@man.ac.uk

This work was supported by the PPP Healthcare Foundation.

(C) 2003 Psychology Press Ltd 
science has been extended to the applied level (e.g., Tallal et al., 1996; Wilson \& Evans, 1996). This latter, applied research, suggests that a particular form of therapy/remediation programme, known as errorless learning, might have benefits over more traditional trial and error methods. The key notion behind errorless learning is that remediation is better if participants are prevented from reinforcing their own errors. Computational neuroscience is beginning to provide a link between clinical application of errorless learning and basic neuroscience. This work has shown that the functioning of neuron-like processing units does not alter if the system reinforces its own errors but change can follow in circumstances like errorless learning (McClelland, Thomas, McCandliss, \& Fiez, 1999).

Errorless learning has a long history. It was first studied and proved effective in the area of animal learning. Under standard procedures, pigeons demonstrate very poor learning of red-green visual discrimination even after repeated exposures. Terrace (1963) found that if an errorless learning method was adopted, pigeons' learning of the same discrimination was very much better. Since then it has been used as a rehabilitation method in a variety of areas. It has been used as a behaviour modification technique with children and adults (Brownjohn, 1988; Cipani \& Spooner, 1997; Ducharme, 1996; Ducharme, Atkinson, \& Poulton, 2000). These methods have been used to teach auditory discrimination skills to children with language-based learning impairments and to children and adults with Down's syndrome, and non-native phonemes to Japanese adults (Brownjohn, 1988; Duffy \& Wishart, 1987, 1994; McCandliss, Fiez, Protopapas, Conway, \& McClelland, 2002; Merzenich et al., 1996; Tallal et al., 1998). It has been used with people with mental illnesses to teach a variety of tasks, including naming (Kern, 1996; O'Carroll, Russell, Lawrie, \& Johnstone, 1999; Wykes et al., 1999). The occupational therapy literature reports it as a technique for dyspraxia intervention (Jackson, 1999). More recently, studies have found that errorless training methods are more successful when teaching golf putting to normal adults (Maxwell, Masters, Kerr, \& Weedon, 2001).

Baddeley (1992) suggested that errorless learning was a possible principle for the practice of memory rehabilitation and it is in the domain of amnesia that much of the recent literature has been focused. Wilson and colleagues have studied the use of errorless learning extensively and compared this with errorful methods. Using this technique they have taught people with amnesia and memory impairment to do a variety of tasks including learning names of objects and people, how to programme an electronic aid, remembering orientation items, and learning items of general knowledge. In each case, errorless learning was superior to errorful learning (Baddeley \& Wilson, 1994; Clare, Wilson, Breen, \& Hodges, 1999; Clare et al., 2000; Evans et al., 2000; Wilson \& Evans, 1996; Wilson, Baddeley, Evans, \& Shiel, 1994). Squires, Hunkin, Parkin and colleagues have also examined errorless and errorful learning. They 
taught amnesic patients word associations, names of objects and people, and basic commands and procedures associated with use of a word processing package (Hunkin, Squires, Parkin, \& Tidy, 1998a; Hunkin, Squires, Aldrich, \& Parkin, 1998b; Parkin, Hunkin, \& Squires, 1998; Squires, Hunkin, \& Parkin, 1997). All showed benefit of errorless over errorful methods.

\section{Definitions}

The key notion behind errorless learning is that for some situations errant behaviour can be self-reinforcing. Therefore, the act of producing an error for a certain stimulus can strengthen this incorrect association such that the error will be even more likely the next time the stimulus is presented. The definition of errorless learning and consequently how this has been applied clinically has varied. O'Carroll et al. (1999, p. 106) described it as, "the elimination of trial and error approaches to learning. In training, subjects begin with very easy discriminations, do not experience failure, and task difficulty is increased extremely gradually". In the occupational therapy literature, where errorless learning is used as a treatment for dyspraxia, the following definition can be found, "learning is more efficient if a trial and error approach is not permitted but the person is given cues or prompts, which achieve success and are gradually withdrawn" (Jackson, 1999, p. 323). Winter and Hunkin attempt to clarify what traditional trial and error approaches are in their definition of errorless learning, "errorless learning is a technique in which the possibility of errors made during training is eliminated. It differs from more traditional forms of learning in which 'guessing' is encouraged and whereby errors may be produced inadvertently" (1999, p. 987). Likewise, Clare et al., (2000, p. 133) describe errorless learning as, “. . . the elimination or reduction of incorrect or inappropriate responses during training...”.

Two generalities emerge from the literature: First errorless learning is not specific to any one type of intervention. It is a characteristic that can be carried across tasks. It is, therefore, not a programme of treatment but a description/approach whereby the task is manipulated to eliminate/reduce errors. Tasks are executed in such a way that the subject is unlikely to make errorsavoiding the typical situation in which patients' errorful responses are corrected by the therapist. This contrasts with traditional approaches where guessing is advocated in the belief that this effortful approach will encourage better performance (O'Carroll et al., 1999).

Second, typically, tasks are gradually made more difficult even at the potential cost of introducing errors into the training. The motivation for increasing task difficulty is twofold: gradual approximation to real life and maintenance of effort/attention during therapy. Errorless learning methods are inherently artificial. Patients are either prevented from making errors by being given the correct response or by making the task so easy that errors are very unlikely. 
Both situations are unlike real language use and this gradual shift away from an errorless technique during therapy is understandable. The second motivation for increasing task difficulty is that errorless paradigms can seem inherently boring. Setting aside the possibility that the therapist but not the patient would lack motivation, this observation raises the question of the relationship between effort and rate of errors. These two factors were recently studied by Komatsu et al. (2000). They suggested that there is an error-effort trade-off and hypothesised that the best condition for learning was one that prevented errors but also required effort. The contrast between effort and rate of errors was studied by experimentally crossing the factors to produce four learning conditions. Eight subjects with severe retrograde amnesia were trained on people's names. They found that the paired associate (errorless and effortless) and vanishing cues (errorless and effortful) methods led to superior recall in comparison to target selection (errorful and effortless) and initial letter (errorful and effortful) methods, demonstrating an overall advantage for errorless learning. In contrast, the effort factor was found to have little effect.

As noted above, a number of reported therapies were designed either to reduce errors without necessarily eliminating them or to relax the task constraints during training thereby allowing errors to creep in. Both approaches raise two key questions. On the assumption that an errorless approach is optimal, (1) how much error can be introduced before the advantage of error reduction is lost, and (2) when is the optimal time to introduce errors during therapy? As far as we are aware there are no human data on these questions. Terrace's (1963) animal study, however, was designed to address these issues. Terrace (1963) taught pigeons to discriminate between red and green. The training regime manipulated the timing (late and early presentation) and frequency (constant and progressive presentation) of error. Terrace found that discrimination accuracy was best for the birds that learnt without being exposed to error, whereas, performance was poorest for birds that made many errors while acquiring the discrimination. Interestingly, he found a type of "age of acquisition" effect. Birds that were prevented from making errors early in the training procedure but then allowed to make errors later were better at discriminating than the birds that were given the reverse training pattern (errors early, errorless late).

These results suggest that learning is best if errors are completely eliminated rather than simply reduced. To draw out this distinction we will adopt the following terms and definitions:

1. Errorless learning - the attempt to control for errors during training. Two training methods can be used:

Error Elimination - no errors are made during training.

Error Reduction - errors are reduced during training.

2. Errorful learning - errors are not controlled during training. 
Although error elimination may be the optimal approach, it should be noted that various practical aspects often prevent studies conducting a completely errorless method. First, most studies do not report if errors were made during training or the pattern of those errors. Second, many studies have tended to assess improvement after every training session. Such assessment is itself a source of errors which, when interleaved with training could reduce or eliminate the advantages of a purely errorless approach (Wilson et al., 1994). Finally, as noted above, because most treatments make the tasks gradually harder this increases the likelihood that errors will be made. Other researchers have also noted that in practice errors sometimes occur during treatment and in testing. Clare et al. (2000), therefore, proposed that the interventions described should be viewed as "approximations of an errorless paradigm" and under the definitions offered above, would be most accurately described as "error reduction" methods.

\section{A theory of errorless learning?}

Hebbian plasticity and Hebbian-based learning rules have guided much of the work in both cortical synaptic plasticity and cortical representational reorganisation. At the synaptic level, Hebbian plasticity refers to increases in synaptic strength between neurons that fire together. At a higher level of neuronal organisation, Hebbian-based learning rules refer to the detection of temporally correlated inputs. A fundamental notion in neuroscience is that synaptic efficiency between two neurons is a substrate for learning and memory (Buonomano \& Merzenich, 1998). One model of errorless learning arises from consideration of the Hebbian learning rule. According to Hebb (1961), if two neurons fire together, the strength of the connection between them will be increased. Therefore, if an input elicits a pattern of neural activity, Hebbian learning will strengthen the tendency to activate the same pattern on subsequent occasions. This means that learning will increase the likelihood of making the same response in the future, whether correct or incorrect (McClelland et al., 1999). Within the neuroscience and psychological literature two closely related theories have emerged to explain why errorless learning can be beneficial. We will review these two theories and then provide our own framework in which to think about errorless and errorful learning.

One approach to understanding the potential benefits of errorless learning, arises from consideration of patients with amnesia. Baddeley and Wilson's (1994) explanation is based on the assumption that there are two types of memory: implicit and explicit. As the terms suggest, implicit memory is subconscious and cannot be deliberately manipulated. Explicit memory has the opposite characteristics. The automatic nature of implicit memory makes it particularly vulnerable to interference from errorful responses. In a similar 
manner to the Hebbian account, implicit memory has no mechanism for filtering out incorrect information. Explicit memory does not suffer from this type of interference because this type of memory is open to conscious manipulation allowing incorrect information to be filtered out. Baddeley and Wilson (1994) argue that amnesic patients have a specific impairment of explicit memory making them particularly vulnerable to interference. Errorless learning is, therefore, optimal for these patients because no memory manipulation or filtering is required.

The issue of whether errorless learning is better characterised by implicit or explicit memory processes has been the centre of some debate (Kalla, Downes, \& van den Broek, 2001). Although maintaining the distinction between implicit and explicit memory, Squires et al. (1997) argued that errorless learning may operate by facilitating either implicit or explicit memory, or both. They found that amnesic patients learned novel associations (unrelated word pairs) better with an errorless than errorful method. Squires et al. (1997) argued that novel association learning is particularly reliant on explicit memory. This suggests that explicit memory might also benefit from errorless techniques. Hunkin et al. (1998a) agreed that residual explicit memory functioning may play a role as they failed to find any correlation between improved errorless learning performance and preserved implicit memory functioning.

O'Carroll et al. (1999) found that errorless learning was also beneficial for patients with schizophrenia. Like Baddeley and Wilson (1994), they suggested that this could be attributed to impaired explicit memory. In an attempt to elucidate the nature of the impairment, O'Carroll et al. (1999) argued that the patients had a source monitoring difficulty. They demonstrated that the patients were unable to determine whether the correct answer was their own selfgenerated incorrect guess or the experimenter's correction.

Irrespective of the exact status of the underlying memory system(s), these studies suggest that there may be some form of cognitive process that can filter out or inhibit the influence of erroneous responses, a mechanism that is absent for amnesic and schizophrenic patients. If we consider results from beyond the amnesia literature, however, it is clear that errorless learning is advantageous for people other than just those with impaired memory. For a complete account, other aspects of the processes that underpin learning (and re-learning) need to be considered. For example, it can be very difficult for adults to learn to perceive certain speech contrasts that their native language does not use. Native Japanese people have persistent difficulty in distinguishing the English liquids [r] and [1]. Even after years of exposure to English, Japanese adults are still markedly poorer at discriminating between these sounds than native English speakers. Recent studies (see below) have been able to remediate these persistent perceptual difficulties using errorless learning. Such demonstrations are important because these participants are entirely neurologically normal and yet errorless learning can induce plasticity in their mature language system. 
McClelland et al. (1999) used a computational model to test the hypothesis that the Japanese adults' poor learning was due to a general learning mechanism that reinforces perceptual categories learnt early in life but prevents new learning. More specifically, Hebbian learning might maintain pre-established perceptual representations even in the face of new experience. McClelland et al. (1999) trained computational, Kohonen networks in English-like and Japanese-like environments. In the English version, the model was trained to discriminate between six different abstract "phonological" representations including $/ 1 /$ and $/ r /$. Tokens of these two phonemes were sampled from two similar but somewhat different distributions. The Japanese version was identical except that the distributions of $/ 1 /$ and $/ \mathrm{r} /$ were identical to mirror the fact that there is no distinction between these two phonemes in the real Japanese language. Following training, the representational layer of the English model had been structured to reflect six different phonemes, including separate spaces for $/ 1 /$ and $/ r /$. Unsurprisingly, the representational space in the Japanese model contained only five distinctions with $/ 1 /$ and $/ \mathrm{r} /$ occupying the same part. In the next, critical step, McClelland et al. (1999) switched the Japanese model to the English-like environment in which tokens of $/ 1 /$ and $/ \mathrm{r} /$ were drawn from overlapping but non-identical distributions. Despite this switch the model, like Japanese people, failed to learn the $/ 1 /-/ \mathrm{r} /$ discrimination. This followed because both $/ 1 /$ and $/ r /$ inputs were captured by the same, single phonological representation, and this incorrect association was continually reinforced by the Hebbian learning used in this model. Although the model, like Japanese people, appeared to be perpetually stuck, McClelland et al. (1999) were able to demonstrate that plasticity and effective learning were possible given the right circumstances. Instead of presenting the model with the standard English-like environment, McClelland et al. (1999) included exaggerated tokens of /1/ and $/ \mathrm{r} /$ thus increasing the separation of their distributions. In these circumstances, the model no longer collapsed $/ \mathrm{l} / \mathrm{and} / \mathrm{r} /$ into the same single representation but altered the representational layer to include six separate phonemes. Most importantly, when the model was returned to the English-like environment with overlapping $/ 1 /$ and $/ \mathrm{r} /$ distributions, it was able to discriminate accurately between the two phonemes.

These simulations provide the obvious prediction that the use of exaggerated contrasts might prove effective for human subjects. Previous work suggests that this type of training regime can be useful for children with language learning impairments (Merzenich et al., 1996; Tallal et al., 1998). McCandliss et al. (2002) conducted an empirical study with Japanese adults in order to test the prediction of the simulations more directly. This study contrasted two important factors: errorless vs. errorful and with feedback vs. without feedback. The errorless condition used an adaptive training method. Japanese subjects began with exaggerated tokens of $/ 1 /$ and $/ \mathrm{r} /$ embedded within a simple word frame (lock and rock). A staircase method was used to gradually 
move the tokens towards those found in normal English. Closer tokens of /1/ and $/ \mathrm{r} /$ were used once the subject had made eight successive correct discriminations while more disparate examples were used if the subject made only one error. This asymmetric staircase method provided an "error reduction" intervention in which errors were deliberately controlled and minimised. In contrast, in the errorful condition the Japanese subjects were presented with standard tokens of $/ 1 /$ and $/ \mathrm{r} /$ throughout the training sessions. These two training regimes were crossed with the feedback factor. Half the subjects were told whether their discrimination choice was correct while the remainder received no feedback. In the no feedback condition, the prediction of the computational model was upheld. The adaptive, error reduction method produced significant improvement in $/ 1 /-/ \mathrm{r} /$ discrimination while the errorful method produced no benefit relative to control. McCandliss et al. (2002) also found that feedback was a critical factor: subjects in the errorful condition who received feedback about their discriminations learned as well as those people in the adaptive, error reducing condition. McCandliss et al. (2002) concluded, therefore, that a model based solely on Hebbian learning (like McClelland et al., 1999) required an additional mechanism to capture the positive effects found in the feedback condition. Two possible mechanisms were proposed: Hebbian learning combined with error-correcting learning methods or Hebbian learning modulated by outcome information as in reinforcement learning.

When this study is taken together with those described above, it is clear that there are a wide range of learning situations and of participants/patients for which errorless learning is beneficial. In addition, the McCandliss et al. (2002) study demonstrates that some subjects are able to use feedback to control for the inherent problems associated with learning in an errorful situation. While amnesia may be an important factor when choosing between an errorful or errorless intervention (Baddeley \& Wilson, 1994), memory impairments do not provide a complete explanation for the whole literature: there are a range of situations in which neurologically intact subjects benefit from an errorless technique (Maxwell et al., 2001; McCandliss et al., 2002). In addition, there are certain circumstances in which even normal subjects with feedback available, still perform better with an errorless method (e.g., when learning a complex motor skill; Maxwell et al., 2001). In an attempt to unify these various findings, we offer the following simple generic framework (see Figure 1).

Following McCelland et al. (1999), we assume that the cognitive representations, which mediate between stimulus and response, are predominantly formed through Hebbian learning. Unlike other learning algorithms, the Hebbian approach allows learning in situations where there is no form of feedback. As noted above, the disadvantage of Hebbian learning is that the system performs poorly in an errorful learning situation. Results like those reported by McCandliss et al. (2002) demonstrate that human cognition is able to use feedback, in some way, to overcome these inherent problems with 


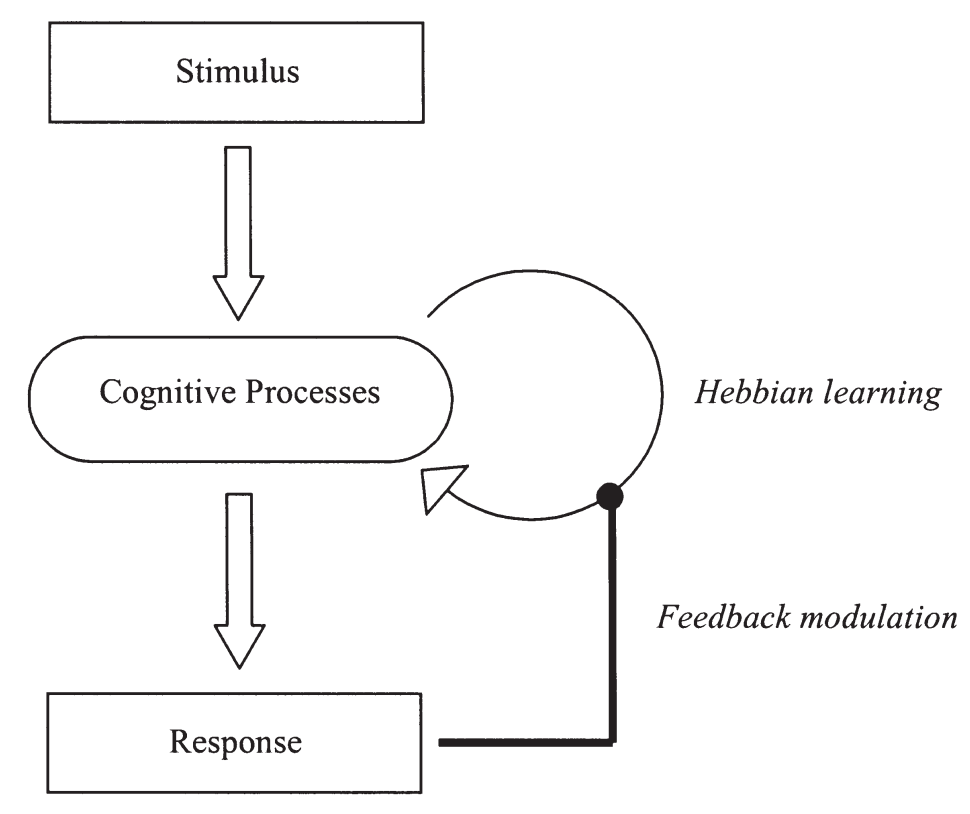

Figure 1. A simple framework for learning in errorful and errorless situations.

Hebbian learning. If this formulation is correct then two key questions arise: What cognitive processes are involved in the feedback modulation of Hebbian learning and in what way is Hebbian learning altered in order to filter out errorful trials? There may be at least three underlying elements to the feedback modulation of the learning mechanism:

1. Before feedback modulation can be initiated, participants must be able to monitor the accuracy of their response.

2. It is possible that feedback modulation necessitates the temporary storage or prolonged activation of the original stimulus and the associated response while the underlying representations are adjusted by the learning mechanism.

3. It is very likely that feedback modulation requires efficient verification and regulation of behaviour and, perhaps, the deliberate manipulation of representations. Such elements call heavily upon attentional and executive resources.

The existing literature contains examples in which each of the three elements of feedback modulation is compromised. In such circumstances, subjects fall back on Hebbian learning and can only improve their performance with an errorless intervention. 
Without the examiner's assistance, for example, normal Japanese subjects have no basis on which to judge their own responses to /1/ and /r/ stimuli (McCandliss et al., 2002). Likewise certain forms of damage to the language system produce the same result. Aphasic patients with semantic damage, for example, cannot tell if their semantic paraphasias are correct or not (Lambon Ralph, Sage, \& Roberts, 2000). In this situation, feedback modulation cannot begin and learning, or relearning, must proceed entirely on a Hebbian basis. On the other hand, if feedback is provided by the examiner, normal feedback modulation can commence and subjects can learn effectively even in an errorful situation (McCandliss et al., 2002). The second underlying element of feedback modulation requires some form of memory (irrespective of the implicit/explicit debate). This would then explain why, even if able to detect the accuracy of their own responses, amnesic patients might fall back on basic Hebbian learning. Third, the deficient source monitoring of patients with schizophrenia provides an example of impaired executive resources (O'Carroll et al., 1999). We note here that although the amnesic patients seem most relevant to the second element of feedback modulation, it is possible that many of the patients in the amnesia literature might also have accompanying attentional-executive disorders. The most common aetiologies represented in this literature, including head injury, dementia of Alzheimer's type, and Herpes Simplex Viral Encephalitis, commonly produce attentional-executive dysfunction in addition to amnesia. Finally, it is possible that the presence of feedback modulation may vary across cognitive domains. Results from studies of complex motor learning (Maxwell et al., 2001) suggest that, even for normal subjects with feedback available, learning in such domains is achieved almost entirely on a Hebbian basis.

The potential modulation of the underlying Hebbian learning process raises the obvious question of how this would be implemented at a computational level. McCandliss et al. (2002) offer two possibilities. The first relates to learning mechanisms in which Hebbian and error-driven learning algorithms are combined (e.g., Hebbian plus backpropogation: O'Reilly, 1996). In this scheme, the Hebbian element allows for learning when there is no form of feedback available. In contrast, if feedback is available, the system can supplement purely Hebbian learning with a deliberate reduction of the difference between the actual response produced and the target required. When the model produces an errorful response, although Hebbian learning will tend to reinforce the association, the error-reduction learning mechanism will alter the underlying representations towards the correct response. In effect, the error-reducing element can filter out the errorful responses that would otherwise be reinforced by purely Hebbian learning. The second possibility is somewhat closer to the processes depicted in Figure 1. In this scheme, learning is based entirely on the Hebbian rule except that, if information about outcome is available, the degree of learning for that trial can be modulated. For example, if a response was 
accurate or produced a positive outcome, Hebbian learning could proceed normally - thus reinforcing the likelihood of producing that response again the next time the stimulus is presented. If the response was incorrect, however, feedback modulation might inhibit the Hebbian process such that little or no learning occurred for that trial. This form of gating would then prevent the system from reinforcing errorful responses.

\section{The use of errorless learning in treating language problems}

The existing literature provides many demonstrations that amnesic subjects are better able to learn new and relearn old information using errorless rather than errorful techniques. The errorless method has been successfully applied to a variety of learning situations including relearning proper nouns and object names. For example, Wilson et al. (1994) taught PS, a patient with dense amnesia following a thalamic stroke, the names of fictitious people using errorful and errorless techniques. Ten first names and 10 surnames were trained with each technique. Three successive learning trials were given for each technique followed by nine test trails, spread over a 30 -min period. In the errorful condition, PS was given the first letter of the name and was asked to guess. If, after three guesses, he was still incorrect then the correct name was given and he was asked to write it down. In the errorless condition, PS was told the first letter, then the name and was asked to write it down. Wilson et al. (1994) found that in eight of the nine test trials, errorless learning resulted in superior learning. Clare et al. (1999) re-taught VJ, a man in the early stages of Alzheimer's disease, the names of 11 members of his social club using an errorless technique. The therapy comprised the following elements: learning a mnemonic for each person; completing the name with a step-by-step reduction of the letters provided (vanishing cues); writing the name by filling in the gaps; recalling the mnemonic used; repeating the name; and finally, recall at short but gradually increasing intervals. The therapy consisted of 21 sessions twice weekly. VJ also practised the same procedure with family members at home three times a day and he was encouraged to name the members while he was at his social club. VJ was tested on the 11 names after each therapy session. These continual assessments showed that the proportion of faces correctly named increased from $22 \%$ at baseline to $98 \%$ following training. Additional testing found that this benefit was maintained after 9 months.

These studies raise the possibility that errorless learning might be a technique that could be used to treat aphasic word finding difficulties (anomia) and, perhaps, a wide variety of acquired language deficits. Studies of children with developmental disorders and normal Japanese adults (McCandliss et al., 2002; Tallal et al., 1998) also indicate that errorless learning methods can be successfully extended from the memory to the language domain. As far as we are 
aware, the aphasia treatment literature contains no studies that have directly investigated the use of errorless learning. A closer look at the literature, however, highlights a number of studies that are comparable to the errorless method. For example, Morris et al. (1996) conducted a study designed to treat word deafness that is analogous to other reports in the errorless learning literature (McCandliss et al., 2002; Tallal et al., 1998). They studied a patient with pre-speech and speech-level perceptual deficits. Using a variety of tasks, Morris et al. (1996) delivered a phonological discrimination therapy in which the patient began with contrasts that varied in three phonetic features (voice, place, and manner of articulation; known as "maximal pairs" tests). Once the patient's performance on this task had improved, he was then moved on to contrasts varying by two features and eventually on to single feature discriminations (known as "minimal pairs" tests). Although not described as such, this staircase method is inherently error reducing and similar in style to the interventions used in the developmental and Japanese studies (McCandliss et al., 2002; Tallal et al., 1998). Morris et al. (1996) found that the patient's discrimination skills improved significantly and had generalised to spoken word recognition and comprehension. Intriguingly, there were some indications that the language-based therapy had also improved the patient's performance on non-verbal auditory discriminations such as gap detection and pitch discrimination.

\section{Review of the anomia treatment literature}

Morris and colleagues' (1996) investigation highlights the fact that although the aphasic therapy literature contains no studies that have set out to examine errorless learning specifically, it does include interventions that either reduce or eliminate errors. Given that the amnesia literature contains many examples of therapies aimed at treating word finding problems, an obvious question is whether errorless techniques might also prove effective for treating anomia in aphasic patients. This would be especially interesting given that word-finding difficulties are perhaps one of the most common and disabling aphasic symptoms. We undertook, therefore, a review of the anomia treatment literature starting from the seminal paper by Howard et al. (1985b). Studies were categorised into one of three classifications noted above: i.e., error elimination, error reducing or errorful. The relative merits of each approach were considered in terms of three efficacy measures-improvement immediately after therapy, residual benefit after a period of no therapy (follow up testing), and generalisation to untreated items. Studies were then split by aphasia type (fluent vs. nonfluent aphasia), principal impairment (expressive vs. both receptive and expressive aphasia), and therapy method (receptive or expressive therapy) to test for variation in outcome for these important factors. It would 
have been interesting to investigate the possibility that concurrent cognitive impairment, e.g., amnesia or executive-attentional deficits, influences the efficacy of errorful and errorless interventions for word finding problems. Unfortunately, too few studies reported sufficient background neuropsychology to be certain about the status of these cognitive systems in the aphasic patients.

\section{Method}

To be included in the review, studies had to conform to the following basic criteria: First, the study must have aimed to treat patients for their word-finding difficulties. A wide variety of patient types were considered as long as they presented with word finding difficulties (i.e., papers from the amnesia, aphasia and other related literatures were included where possible). Studies that indirectly improved naming as a by-product of another therapy (e.g., therapy to improve comprehension) were excluded. Second, all interventions had to fall under the classification of cognitive-behavioural remediation. Other interventions such as drug studies were excluded. Third, the study's method had to be explicitly described such that the therapy could be repeated by other researchers. The fourth criterion required the study to have completed baseline or multiple baseline measures of naming therapy-targeted and control items. Fifth, the study had to test the naming accuracy of the treated items pre- and post-therapy rather than using a generic naming test which did not include the treated items. Sixth, it had to include a set of control items to be able to confirm a specific therapy effect. Seventh, any changes in performance had to be confirmed by appropriate statistical analysis (e.g., McNemar change test). Finally, no other therapy should have been carried out during the study. Although we were interested in both immediate and longer-term effects of therapy, follow up assessment of treated and untreated items was not included as a formal criterion of inclusion.

The form of the studies typically conducted in the amnesia literature meant that only 1/11 studies were included in the review: 10 studies were excluded because the assessment did not include a set of control items. From the aphasia treatment literature, 52 articles were considered and 25 were excluded. Reasons for exclusion included the following - nine articles failed to include statistical analysis, important details of the therapy method were omitted from seven studies, three investigated the effect of cueing rather than therapy, multiple simultaneous therapies were conducted in two articles, control items were omitted from two papers, and another two studies used a functional therapy approach.

Information about each study is summarised in Table 1. Each row represents one type of treatment. Studies sometimes included a number of different treatments. Each of these was considered separately and is represented on multiple 


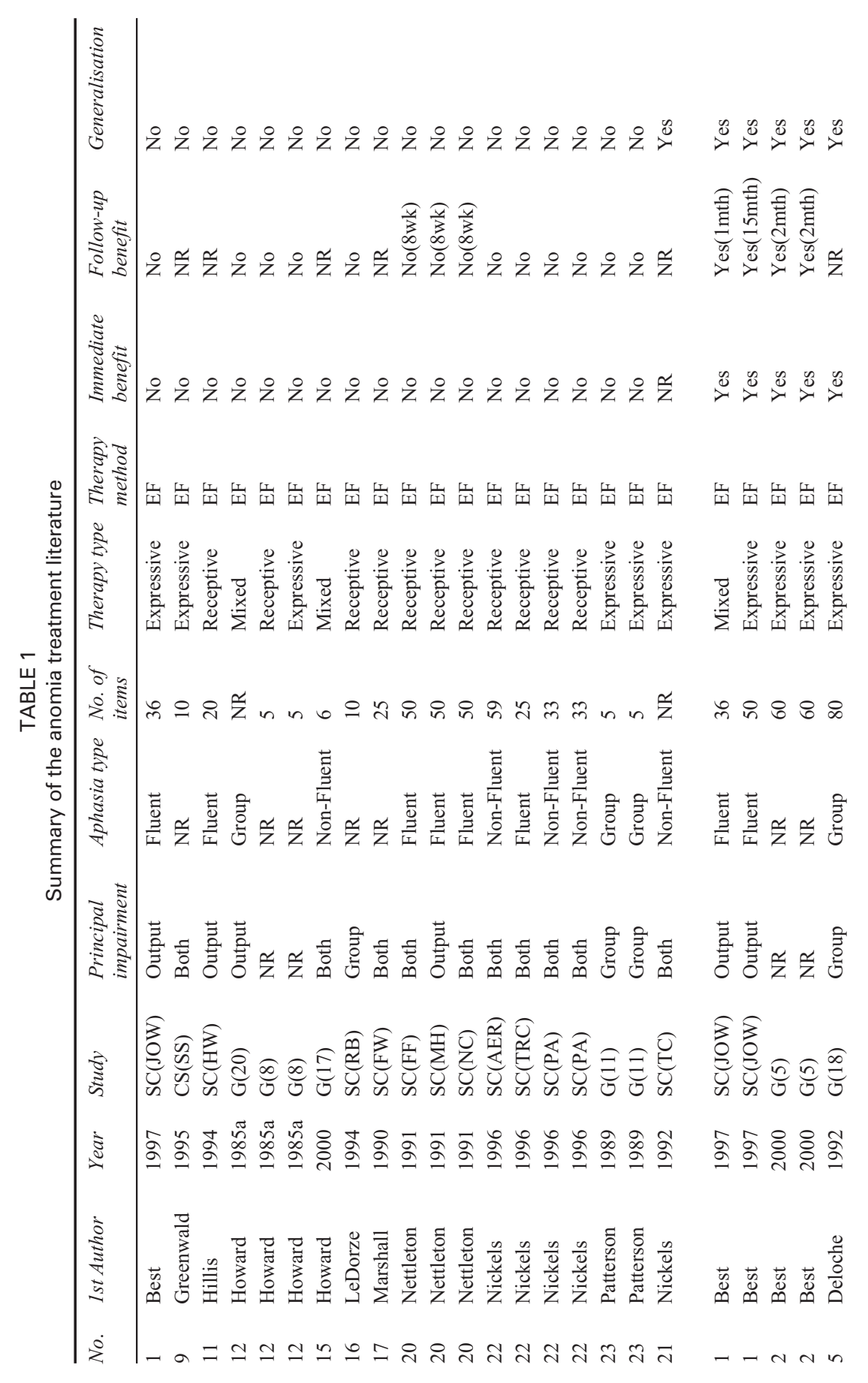




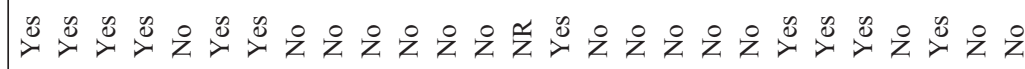

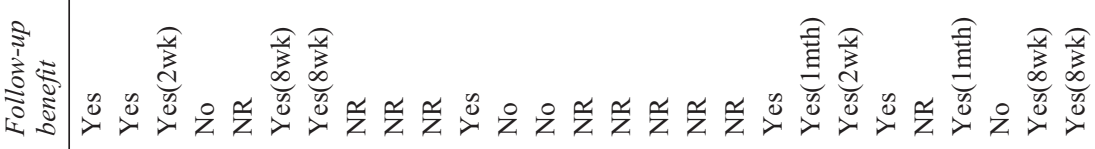

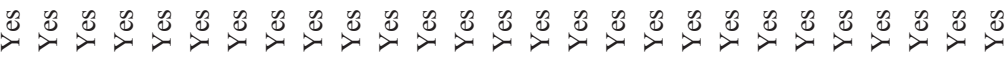

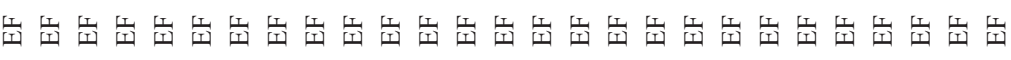

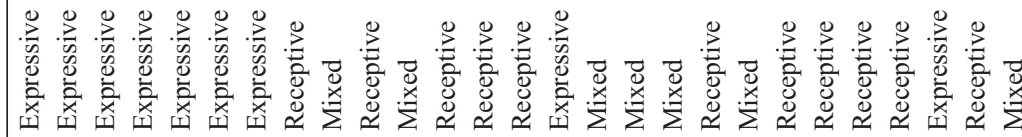

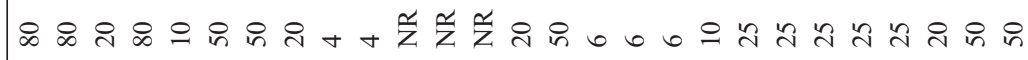
בั

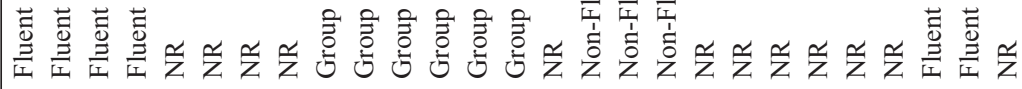

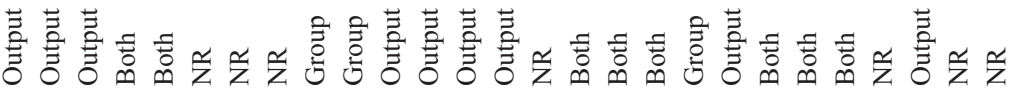

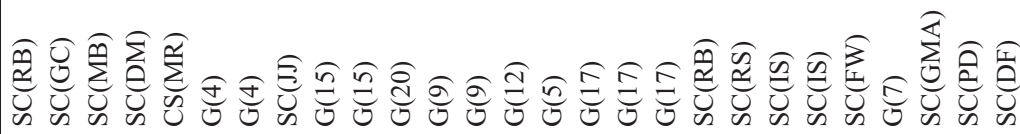

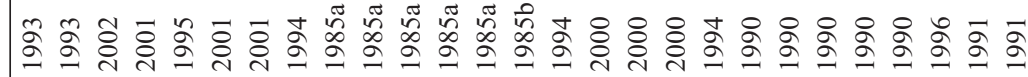

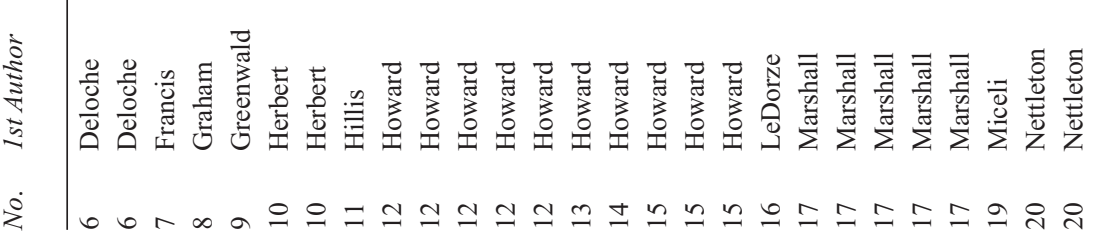




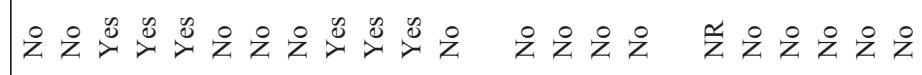

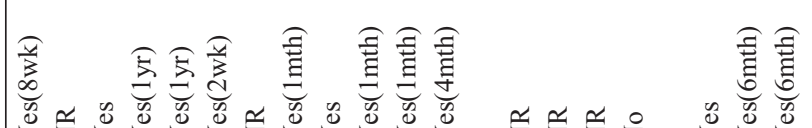

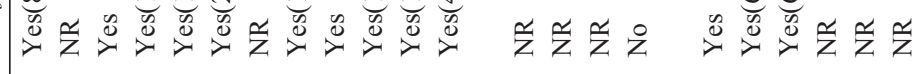

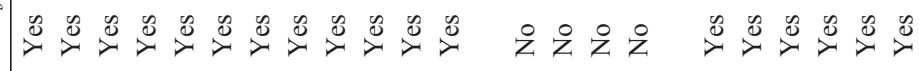

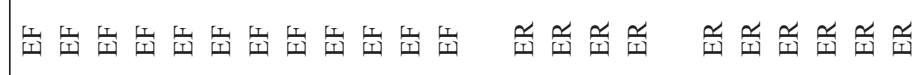

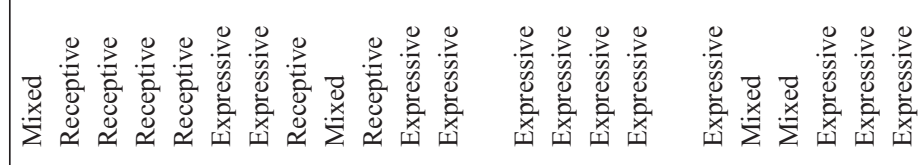

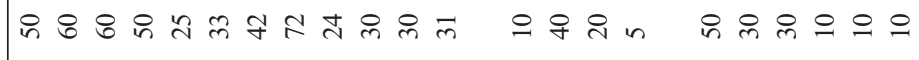

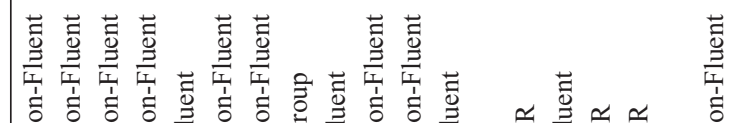

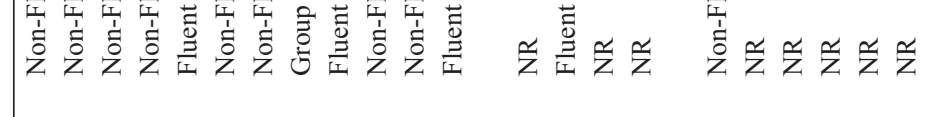

ธ

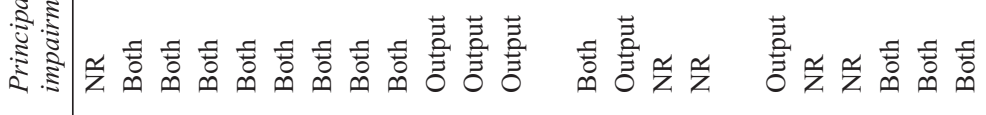

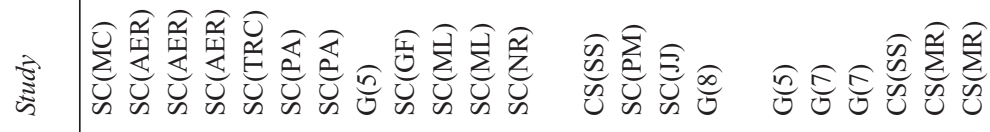

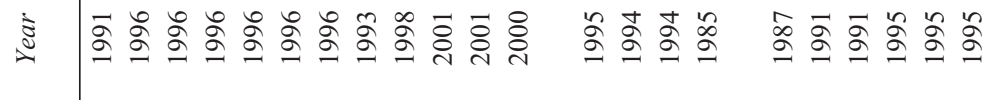

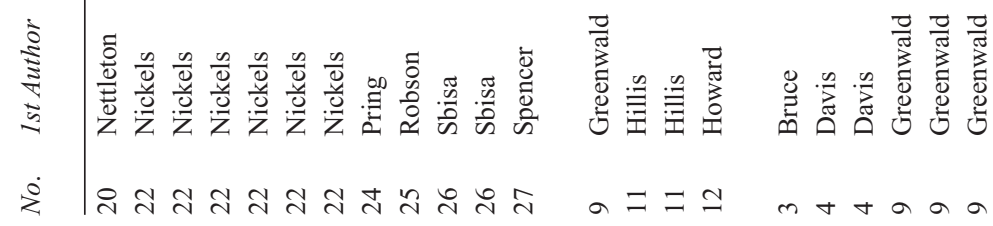




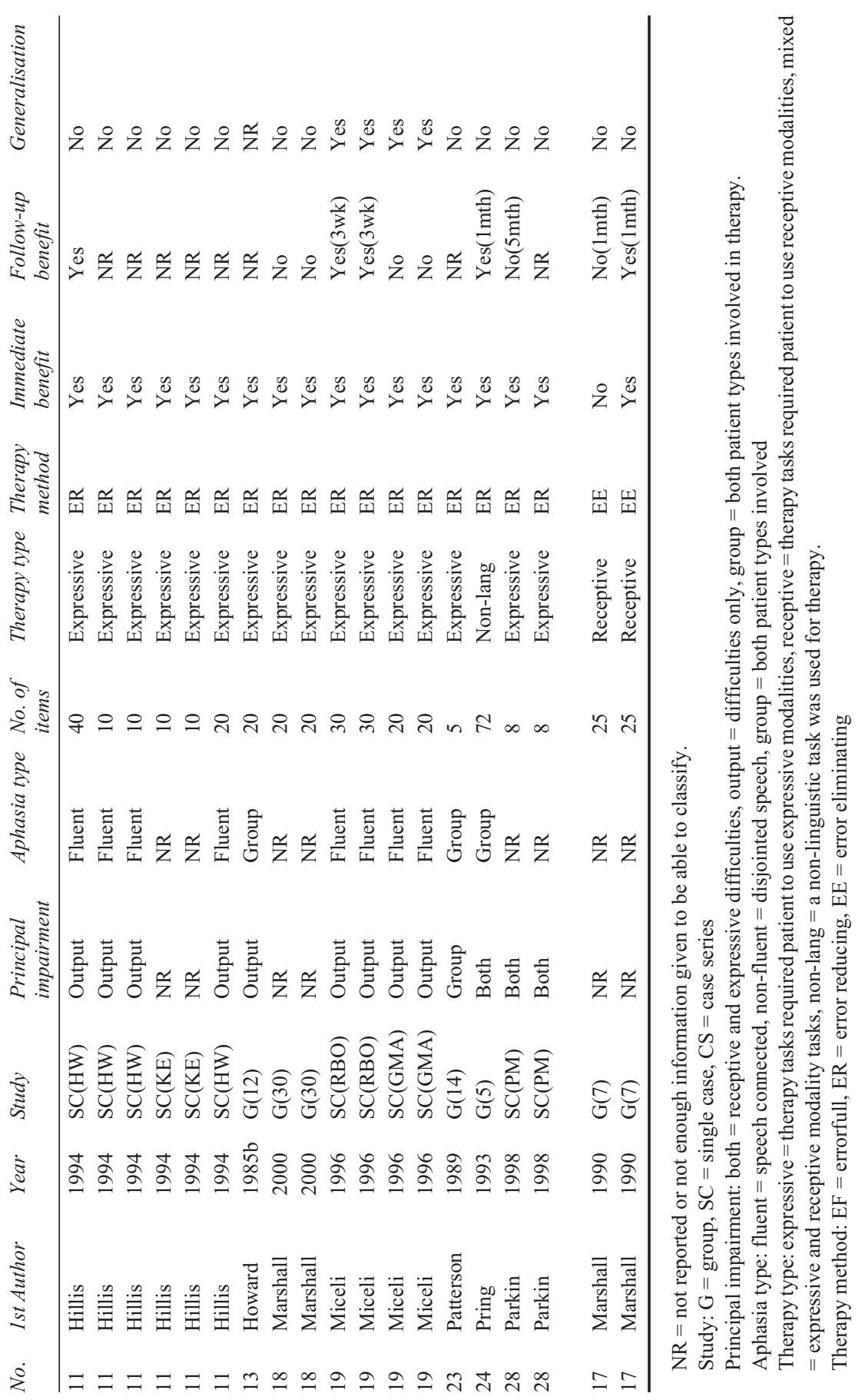


rows, one for each treatment type. So, for example, study number 1 (Best, Howard, Bruce, \& Gatehouse, 1997) contained information about three different treatments for patient JOW, and each of these is summarised separately in three rows. Likewise, if studies included a case series of patients, information about each patient is divided into separate rows. Group studies are summarised in a single row. The study type, single case or group, is indicated in the fourth column along with either the patient's initials or the number of patients included in the group study. The principal impairmenteither expressive aphasia or combined receptive and expressive deficits-is noted in column five. The aphasia type-fluent or nonfluent-is summarised in the sixth column. Authors' own classifications were used, or if absent, information from the case descriptions were used to categorise the patients. For articles where insufficient information was given, no classification was made and "not reported" (NR) was entered into the Table. The Table also includes information about the number of items included in the treatment set, the therapy type (receptive, e.g., word-picture matching, or expressive therapies, e.g., naming with reading aloud) and therapy method (errorful, error reducing, and error elimination). Outcome immediately after therapy, at follow up and generalisation to control items, is noted for each study. Information in the Table is sorted first by therapy method (errorful, error reducing, and then error elimination), then by outcome immediately after therapy (no improvement vs. significant therapy effect) and finally by study number.

\section{Results}

The anomia review shows that out of the 92 therapy interventions, 61 used an errorful learning technique whereas 31 used an errorless method of which 29 were error reducing and 2 were error eliminating. An equal number of errorful (72\%) and error reducing (79\%) studies showed significant improvement immediately after therapy. At follow-up, 59\% of errorful and $47 \%$ of error reducing treatments had a significant lasting effect. There was successful generalisation to a control set of items for $15 \%$ of error reducing and $38 \%$ of errorful techniques. Of the two error elimination treatments, one was successful immediately post-training and at follow-up, but did not generalise to untreated items. Table 1 also shows that the number of items used in therapy does not appear to be a factor that governs type of therapy, technique, or success.

Table 2 summarises the results for each technique (errorful, error reducing, error elimination) by therapy type (expressive, receptive, mixed, nonlanguage). It shows the number of treatments in each category and the rate of success in respect of three efficacy measures: immediately post-therapy, at a follow-up assessment, and generalisation to an untreated set of control items. It should be noted that many studies did not report outcome at follow-up or about 
TABLE 2

Number of studies and success rate by therapy type

\begin{tabular}{|c|c|c|c|c|}
\hline \multirow[b]{2}{*}{ Therapy type } & & \multicolumn{3}{|c|}{ Type of Technique } \\
\hline & & Errorful & Error reducing & Error elimination \\
\hline \multirow{4}{*}{$\begin{array}{l}\text { Expressive } \\
\text { Rate of success }\end{array}$} & $N=47$ & $N=24$ & $N=23$ & $N=0$ \\
\hline & Immediately & $17 / 23(74 \%)$ & $20 / 23(87 \%)$ & $0 / 0$ \\
\hline & Follow-up & $11 / 18(61 \%)$ & 4/9 (44\%) & $0 / 0$ \\
\hline & Generalisation & $14 / 24(58 \%)$ & $4 / 9(44 \%)$ & $0 / 0$ \\
\hline \multirow{4}{*}{$\begin{array}{l}\text { Receptive } \\
\quad \text { Rate of success }\end{array}$} & $N=30$ & $N=28$ & $N=0$ & $N=2$ \\
\hline & Immediately & $17 / 28(61 \%)$ & $0 / 0$ & $1 / 2(50 \%)$ \\
\hline & Follow-up & $10 / 21(48 \%)$ & $0 / 0$ & $1 / 2(50 \%)$ \\
\hline & Generalisation & $7 / 27(26 \%)$ & $0 / 0$ & $0 / 0$ \\
\hline \multirow{4}{*}{$\begin{array}{l}\text { Mixed } \\
\quad \text { Rate of success }\end{array}$} & $N=14$ & $N=14$ & $N=2$ & $N=0$ \\
\hline & Immediately & $10 / 14(71 \%)$ & $2 / 2(100 \%)$ & $0 / 0$ \\
\hline & Follow-up & $6 / 9(67 \%)$ & $2 / 2(100 \%)$ & $0 / 0$ \\
\hline & Generalisation & $2 / 14(14 \%)$ & $0 / 0$ & $0 / 0$ \\
\hline \multirow{4}{*}{$\begin{array}{l}\text { Non-language } \\
\text { Rate of success }\end{array}$} & $N=1$ & $N=0$ & $N=1$ & $N=0$ \\
\hline & Immediately & $0 / 0$ & $1 / 1(100 \%)$ & $0 / 0$ \\
\hline & Follow-up & $0 / 0$ & $1 / 1(100 \%)$ & $0 / 0$ \\
\hline & Generalisation & $0 / 0$ & $0 / 0$ & $0 / 0$ \\
\hline \multirow{4}{*}{$\begin{array}{l}\text { Overall } \\
\quad \text { Rate of success }\end{array}$} & $N=92$ & $N=66$ & $N=26$ & $N=2$ \\
\hline & Immediately & $44 / 65(68 \%)$ & $23 / 26(88 \%)$ & $1 / 2(50 \%)$ \\
\hline & Follow-up & $27 / 48(56 \%)$ & $7 / 12(58 \%)$ & $1 / 2(50 \%)$ \\
\hline & Generalisation & $23 / 65(36 \%)$ & $4 / 9(44 \%)$ & $0 / 0$ \\
\hline
\end{tabular}

Expressive; therapy tasks required patient to use expressive modalities, Receptive; therapy tasks required patient to use receptive modalities, Mixed; expressive and receptive modality tasks, Nonlanguage; a non-linguistic task was used for therapy.

generalisation, therefore, the absolute number of studies included in the calculations for rate of success vary. Expressive therapies were equally distributed between errorful (24) and error reducing (23), techniques. Of the receptive therapies reported, however, none used an error reducing technique, only two used an error eliminating method and 28 used an errorful technique. For mixed therapy, the majority (14) used an errorful technique and only two used an error reducing method. The one non-language type therapy used an error reducing technique. It is, therefore, only for expressive type therapies that the number of error reducing and errorful treatments is sufficient to compare the rate of success. Immediately post training, $74 \%$ of errorful and $87 \%$ of error reducing 
techniques were successful. At follow-up and for generalisation, the errorful techniques have a numerical advantage $61 \%$ vs. $44 \%$ and $58 \%$ vs $44 \%$, respectively (although none is significantly different).

Table 3 splits the errorful and error reducing studies by principal impairment, where patients are classified according to whether they had an expressive impairment only or both receptive and expressive difficulties. It shows the number of studies falling into each category and the rate of success with respect to immediate and long-term improvement and generalisation to untreated items. Table 3 excludes 22 therapies because they did not report the type of patient involved or there was not enough information to classify the patients. Of these 22, 12 used errorful, 8 used error reducing, and 2 used error eliminating techniques. It also excludes eight therapies (seven errorful and one error reducing technique) because these were group studies that involved patients of both categories.

There was an equal distribution between patients with expressive difficulties only (29), and those with both receptive and expressive difficulties (33). As found previously, there was a greater number of studies that used errorful techniques. The proportion of studies that had a significant immediate effect was similar across impairment types. There was a $91 \%$ success rate for error reducing techniques for patients with expressive difficulties and $86 \%$ for patients with both difficulties. For errorful techniques, $78 \%$ of studies

TABLE 3

Number of studies and success rate by principal impairment

\begin{tabular}{|c|c|c|c|}
\hline \multirow[b]{2}{*}{ Principal impairment } & & \multicolumn{2}{|c|}{ Type of technique } \\
\hline & & Errorful & Error reducing \\
\hline Expressive & $N=29$ & $N=18$ & $N=11$ \\
\hline \multirow[t]{3}{*}{ Rate of success } & Immediately & $14 / 18(78 \%)$ & 10/11 (91\%) \\
\hline & Follow-up & $10 / 16(62 \%)$ & $4 / 6(66 \%)$ \\
\hline & Generalisation & $8 / 17(47 \%)$ & $4 / 9(44 \%)$ \\
\hline Receptive and expressive & $N=33$ & $N=26$ & $N=7$ \\
\hline \multirow[t]{3}{*}{ Rate of success } & Immediately & $16 / 25(64 \%)$ & $6 / 7(86 \%)$ \\
\hline & Follow-up & $8 / 15(53 \%)$ & $1 / 2(50 \%)$ \\
\hline & Generalisation & $9 / 26(35 \%)$ & $0 / 7$ \\
\hline Overall & $N=62$ & $N=44$ & $N=18$ \\
\hline \multirow[t]{3}{*}{ Rate of success } & Immediately & $30 / 43(70 \%)$ & 16/18 (89\%) \\
\hline & Follow-up & $18 / 31(58 \%)$ & $5 / 8(63 \%)$ \\
\hline & Generalisation & $17 / 43(40 \%)$ & $4 / 16(25 \%$ \\
\hline
\end{tabular}

Fluent; speech is connected, Non-fluent; disjointed speech. 
exhibited an immediate effect for expressive patients and $64 \%$ for patients with both expressive and receptive difficulties. When using an errorful technique for patients with output difficulties, $62 \%$ had lasting effects and $47 \%$ generalised to untreated control items. In contrast, using an errorful technique for patients with both receptive and expressive difficulties, 53\% had lasting effects and $35 \%$ generalised. When an error reducing technique was used for patients who had output difficulties, $66 \%$ had lasting effects and $44 \%$ generalised to an untreated set of control items. There were few studies that used an error reducing technique for patients with both expressive and receptive difficulties, however, one of two studies found a lasting effect and none generalised.

Table 4 splits the studies in terms of the patients' fluency. Thirty-five therapies are excluded because they did not report or do not have enough information to classify the patients' fluency. An additional 14 group studies are removed because they included both fluent and non-fluent patients. This leaves limited numbers to comment on. However, there continues to be a similar rate of success for both techniques immediately post-training, at follow-up, and generalisation. For error reducing techniques immediately post-training, $89 \%$ were successful with fluent patients, and for errorful techniques with the same patient type, $65 \%$ were successful. When follow-up and generalisation are considered, both techniques appear to be equally likely to produce a positive outcome. Very few studies have used errorless techniques with non-fluent patients.

TABLE 4

Number of studies and success rate by patient type

\begin{tabular}{llll}
\hline \multirow{2}{*}{ Patient type } & & \multicolumn{2}{c}{ Type of technique } \\
\cline { 3 - 3 } Fluent & & Errorful & Error reducing \\
Rate of success & $N=26$ & $N=17$ & $N=9$ \\
& Immediately & $11 / 17(65 \%)$ & $8 / 9(89 \%)$ \\
& Follow-up & $9 / 16(56 \%)$ & $3 / 5(60 \%)$ \\
& Generalisation & $9 / 17(35 \%)$ & $4 / 9(44 \%)$ \\
Non-fluent & $N=17$ & $N=16$ & $N=1$ \\
Rate of success & Immediately & $11 / 15(73 \%)$ & $1 / 1(100 \%)$ \\
& Follow-up & $6 / 9(67 \%)$ & $1 / 1(100 \%)$ \\
& Generalisation & $5 / 16(31 \%)$ & $0 / 1$ \\
Overall & & & \\
Rate of success & $N=43$ & $N=33$ & $N=10$ \\
& Immediately & $22 / 32(69 \%)$ & $9 / 10(90 \%)$ \\
& Follow-up & $15 / 25(60 \%)$ & $4 / 6(67 \%)$ \\
& Generalisation & $11 / 33(33 \%)$ & $4 / 10(40 \%)$ \\
\hline
\end{tabular}

Fluent; speech is connected, Non-fluent; disjointed speech. 


\section{Discussion}

The review of the anomia treatment literature highlighted a reasonable number of studies that can be classified as error reducing. Although the number of therapies utilising errorful techniques outweighs those with some form of errorless learning, the rate of success was equivalent for both types of therapy. Although the number of studies prevents formal statistical analysis, the review found evidence to suggest that errorless approaches are just as likely to achieve a positive outcome (in terms of immediate effect, follow-up testing and generalisation). This did not seem to vary if the studies were split by therapy type, principal impairment or patient type. There were, however, a number of areas where there is very little information. For example, there are very few examples of errorless learning for treating anomia with a receptive technique. Likewise there was only one study that used an error reducing approach with a nonfluent patient. It is also notable that many errorless learning studies did not report long-term effects and generalisation.

It is not surprising to find that only two therapies used the potentially optimal, error eliminating approach. This may be because articles do not report error rate or it may be that, in practice, it is extremely difficult to prevent people from making mistakes. The review has shown that error reducing techniques do have positive effects for patients with word finding difficulties. As yet there is limited information on which to judge whether this technique is significantly advantageous over errorful approaches for treating word finding difficultiesalthough the review has shown that error reducing techniques are at least as good as the more traditional trial and error interventions. In addition, very little is known about the effects of error reducing methods on untreated items and whether there is a lasting effect of training because very few studies have included the necessary measures.

\section{CONCLUSION}

In this review we have attempted to describe the basic ideas behind errorless learning. The use of errorless learning has been successfully applied to a variety of groups including normal neurologically intact adults, children with learning difficulties and patients with amnesia following different forms of brain damage. Despite the fact that this literature has used errorless techniques in the domain of language, there have been few, if any, studies of patients with aphasia that have deliberately investigated errorless learning techniques. A review of the anomia treatment literature since 1985 did, however, highlight a small but reasonable number of studies that have used interventions that can be classified as error reducing. When compared with techniques that are inherently errorful in nature, we found that both methods appear to be equally likely 
to produce a positive outcome, not only immediately after therapy but also at follow-up and in terms of generalisation to untreated items.

Errorless learning is a particularly interesting topic because it bridges the gap between practical rehabilitation and basic science from neuropsychology and neuroscience. A number of theories of errorless learning and its relationship to issues of plasticity can be found in the literature. These include Wilson and colleagues explanation in terms of implicit and explicit memory systems as well as McClelland et al.'s computational model of phoneme discrimination learning (McClelland et al., 1999; Wilson et al., 1994). In an attempt to draw these separate literatures and theories together, we have offered a generic framework in which one can consider learning and relearning. The assumptions of this framework are that the basic underlying learning mechanism is Hebbian in nature. While this allows the system to learn when no feedback is available, it does make the process sensitive to errorful trials during learning. For most learning situations, however, the functioning of this simple learning device can be modulated by a feedback system. This feedback modulation can only be executed if information about the accuracy of response is available to the learner. In addition, the feedback system is probably underpinned by at least two generic cognitive systems: memory and attentional-executive processes. If any of these elements are compromised, e.g., after brain damage, learning will tend to revert to a simple Hebbian mechanism. In such circumstances, errorless techniques will be preferable.

While much of this generic framework is based on findings from the literature, future studies will need to test the specific aspects in more detail. In particular, there is a striking lack of information about the application of errorless learning to the treatment of aphasic disorders. Although one can glean some basic indications from the current literature, many questions remain unanswered. Future treatment studies of patients with aphasia are needed to test the relative efficacy of errorful and errorless techniques, perhaps through a withinsubjects design. In addition, error elimination and error reducing techniques could be contrasted. If the relative efficacy of errorful and errorless methods does vary across patients, it will be important to understand whether this relates to the pattern of underlying language and cognitive deficits in these cases. Specifically, it will be interesting to know whether errorless methods are preferable in situations where the feedback system appears to be compromised (e.g., the aphasic patients have amnesia or attentional-executive deficits). Although our literature review deliberately focused on the treatment of word finding difficulties, it is possible that other language deficits might also respond well to an errorless approach (e.g., auditory comprehension: Morris et al., 1996). Finally, on a more pragmatic note, studies might also investigate whether it is realistic to offer errorless learning within the constraints of standard clinical practice. The studies of amnesic patients have tended to use a protocol that is intensive in terms of both patient and staff time. It will be 
important to establish that errorless learning can be delivered effectively within the limited number of therapy sessions which the modern speech and language therapy service is able to support.

\section{REFERENCES}

Baddeley, A. D. (1992). Implicit memory and errorless learning: A link between cognitive theory and neuropsychological rehabilitation? In L. R. Squire \& N. Butters (Eds.), Neuropsychology of memory (2nd ed., pp. 309-314). New York: Guilford Press.

Baddeley, A., \& Wilson, B. A. (1994). When implicit learning fails: Amnesia and the problem of error elimination. Neuropsychologia, 32(1), 53-68.

Best, W., Hickin, J., Herbert, R., Howard, D., \& Osborne, F. (2000). Phonological facilitation of aphasic naming and predicting the outcome of treatment for anomia. Brain and Language, 74(3), 435-438

Best, W., Howard, D., Bruce, C., \& Gatehouse, C. (1997). Cueing the words: A single case study of treatments for anomia. Neuropsychological Rehabilitation, 7(2), 105-141.

Brownjohn, M. D. (1988). Acquisition of Makaton symbols by a young man with severe learning difficulties. Behavioural Psychotherapy, 16(2), 85-94.

Bruce, C., \& Howard, D. (1987). Computer-generated phonemic cues: An effective aid for naming in aphasia. British Journal of Disorders of Communication, 22, 191-201.

Buonomano, D. V., \& Merzenich, M. M. (1998). Cortical plasticity: From synapses to maps. Annual Review of Neuroscience, 21, 149-186.

Cipani, E., \& Spooner, F. (1997). Treating problem behaviors maintained by negative reinforcement. Research in Developmental Disabilities, 18(5), 329-342.

Clare, L., Wilson, B. A., Breen, K., \& Hodges, J. R. (1999). Errorless learning of face-name associations in early Alzheimer's disease. Neurocase, 5(1), 37-46.

Clare, L., Wilson, B. A., Carter, G., Breen, K., Gosses, A., \& Hodges, J. R. (2000). Intervening with everyday memory problems in dementia of Alzheimer type: An errorless learning approach. Journal of Clinical \& Experimental Neuropsychology, 22(1), 132-146.

Davis, A., \& Pring, T. (1991). Therapy for word-finding deficits: More on the effects of semantic and phonological approaches to treatment with dysphasic patients. Neuropsychological Rehabilitation, 1(2), 135-145.

Deloche, G. (1992). Confrontation naming rehabilitation in aphasics: A computerised written technique. Neuropsychological Rehabilitation, 2, 117-124.

Deloche, G., Dordain, M., \& Kremin, H. (1993). Rehabilitation of confrontation naming in aphasia: Relations between oral and written modalities. Aphasiology, 7(2), 201-216.

Ducharme, J. M. (1996). Errorless compliance training: Optimizing clinical efficacy. Behavior Modification, 20(3), 259-280.

Ducharme, J. M., Atkinson, L., \& Poulton, L. (2000). Success-based, noncoercive treatment of oppositional behavior in children from violent homes. Journal of the American Academy of Child and Adolescent Psychiatry, 39(8), 995-1004.

Duffy, L., \& Wishart, J. G. (1987). A comparison of two procedures for teaching discrimination skills to Down's Syndrome and non-handicapped children. British Journal of Educational Psychology, 57(3), 265-278.

Duffy, L. A., \& Wishart, J. G. (1994). The stability and transferability of errorless learning in children with Down's syndrome. Down Syndrome: Research \& Practice, 2(2), 51-58.

Evans, J. J., Wilson, B. A., Schuri, U., Andrade, J., Baddeley, A., Bruna, O., Canavan, T., Della Sala, S., Green, R., Laaksonen, R., Lorenzi, L., \& Taussik, I. (2000). A comparison of "errorless" and "trial-and-error" learning methods for teaching individuals with acquired memory deficits. Neuropsychological Rehabilitation, 10(1), 67-101. 
Francis, D. R., Clark, N., \& Humphreys, G. W. (2002). Circumlocution-induced naming (CIN): A treatment for effecting generalisation in anomia? Aphasiology, 16(3), 243-259.

Graham, K. S., Patterson, K., Pratt, K. H., \& Hodges, J. R. (2001). Can repeated exposure to "forgotten" vocabulary help alleviate word-finding difficulties in semantic dementia? An illustrative case study. Neuropsychological Rehabilitation, 11(3-4), 429-454.

Greenwald, M. L., Raymer, A. M., Richardson, M. E., \& Rothi, L. J. G. (1995). Contrasting treatments for severe impairments of picture naming. Neuropsychological Rehabilitation, $5(1-2), 17-49$.

Hebb, D. O. (1961). The organization of behaviour; A neuropsychological theory. Stimulus and response - and what occurs in the brain in the interval between them. New York: Science Editions, Inc.

Herbert, R., Best, W., Hickin, J., Howard, D., \& Osborne, F. (2001). Phonological and orthographic approaches to the treatment of word retrieval in aphasia. International Journal of Language \& Communication Disorders, 36(2), 7-12.

Hillis, A. E., \& Caramazza, A. (1994). Theories of lexical processing and rehabilitation of lexical deficits. In M. J. Riddoch \& G. W. Humphreys (Eds.), Cognitive Neuropsychology and Cognitive Rehabilitation. Hove, UK: Lawrence Erlbaum Associates Ltd.

Howard, D. (1994). The treatment of acquired aphasia. Philosophical Transactions of the Royal Society of London Series B-Biological Sciences, 346(1315), 113-120.

Howard, D., Best, W., Clark, P., Hickin, J., \& Redmond, T. (2000). Facilitation of word retrieval in aphasia revisited. Brain and Language, 74(3), 441-444.

Howard, D., Patterson, K., Franklin, S., Orchard Lisle, V., \& Morton, J. (1985a). The facilitation of picture naming in aphasia. Cognitive Neuropsychology, 2(1), 49-80.

Howard, D., Patterson, K., Franklin, S., Orchard Lisle, V., \& Morton, J. (1985b). Treatment of word retrieval deficits in aphasia: A comparison of two therapy methods. Brain, 108, $817-829$.

Hunkin, N. M., Squires, E. J., Aldrich, F. K., \& Parkin, A. J. (1998b). Errorless learning and the acquisition of word processing skills. Neuropsychological Rehabilitation, 8(4), 433-449.

Hunkin, N., Squires, E., Parkin, A., \& Tidy, J. (1998a). Are the benefits of errorless learning dependent on implicit memory? Neuropsychologia, 36(1), 25-36.

Jackson, T. (1999). Dyspraxia: Guidelines for intervention. British Journal of Occupational Therapy, 62(7), 321-326.

Kalla, T., Downes, J. J., \& van den Broek, M. (2001). The pre-exposure technique: Enhancing the effects of errorless learning in the acquisition of face-name associations. Neuropsychological Rehabilitation, 11(1), 1-16.

Kern, R. (1996). Cognitive rehabilitation of people with mental illness. Psychiatric Rehabilitation Skills, 1(2), 65-73.

Komatsu, S., Mimura, M., Kato, M., Wakamatsu, N., \& Kashima, H. (2000). Errorless and effortful processes involved in the learning of face-name associations by patients with alcoholic Korsakoff's syndrome. Neuropsychological Rehabilitation, 10(2), 113-132.

Lambon Ralph, M. A., Sage, K., \& Roberts, J. (2000). Classical anomia: A neuropsychological perspective on speech production. Neuropsychologia, 38(2), 186-202.

Le Dorze, G., Boulay, N., Gaudreau, J., \& Brassard, C. (1994). The contrasting effects of a semantic versus a formal-semantic technique for the facilitation of naming in a case of anomia. Aphasiology, 8(2), 127-141.

Marshall, J., Pound, C., White-Thompson, M., \& Pring, T. (1990). The use of picture/word matching tasks to assist word retrieval in aphasic patients. Aphasiology, 4(2), 167-184.

Marshall, R. C., Karow, C. M., Freed, D. B., \& Babcock, P. (2000). Learning of subordinate category names by aphasic subjects: Phonological versus personalized cueing. Brain and Language, 74(3), 438-441.

Maxwell, J. P., Masters, R. S. W., Kerr, E., \& Weedon, E. (2001). The implicit benefit of learning without errors. The Quarterly Journal of Experimental Psychology, 54(4), 1049-1068. 
McCandliss, B., Fiez, J., Protopapas, A., Conway, M., \& McClelland, J. (2002). Success and failure in teaching the $/ \mathrm{r} /-/ 1 /$ contrast to Japanese adults: Tests of a Hebbian model of plasticity and stabilization in spoken language perception. Cognitive, Affective, \& Behavioural Neuroscience, 2(2), 89-108.

McClelland, J., Thomas, A. G., McCandliss, B., \& Fiez, J. (1999). Understanding failures of learning: Hebbian learning, competition for representational space, and some preliminary data. Progress in Brain Research, 121, 75-80.

Merzenich, M., Jenkins, W., Johnston, P., Schreiner, C., Miller, S., \& Tallal, P. (1996). Temporal processing deficits of language-learning impaired children ameliorated by training. Science, 271, 77-84.

Miceli, G., Amitrano, A., Capasso, R., \& Caramazza, A. (1996). The treatment of anomia resulting from output lexical damage: Analysis of two cases. Brain \& Language, 52(1), $150-174$.

Morris, J., Franklin, S., Ellis, A. W., Turner, J. E., \& Bailey, P. J. (1996). Remediating a speech perception deficit in an aphasic patient. Aphasiology, 10(2), 137-158.

Nettleton, J., \& Lesser, R. (1991). Therapy for naming difficulties in aphasia: Application of a cognitive neuropsychological model. Journal of Neurolinguistics, 6, 139-157.

Nickels, L. (1992). The autocue? Self-generated phonemic cues in the treatment of a disorder of reading and naming. Cognitive Neuropsychology, 9(2), 155-182.

Nickels, L., \& Best, W. (1996). Therapy for naming disorders (Part II): Specifics, surprises and suggestions. Aphasiology, 10(2), 109-136.

O’Carroll, R. E., Russell, H. H., Lawrie, S. M., \& Johnstone, E. C. (1999). Errorless learning and the cognitive rehabilitation of memory-impaired schizophrenic patients. Psychological Medicine, 29(1), 105-112.

O'Reilly, R. C. (1996). Biologically plausible error-driven learning using local activation differences: The generalized recirculation algorithm. Neural Computation, 8, 895-938.

Parkin, A. J., Hunkin, N. M., \& Squires, E. J. (1998). Unlearning John Major: The use of errorless learning in the reacquisition of proper names following herpes simplex encephalitis. Cognitive Neuropsychology, 15(4), 361-375.

Patterson, K., Purell, C., \& Morton, J. (1989). Facilitation of word retrieval in aphasia. In C. Code \& D. J. Muller (Eds.), Aphasia therapy: Studies in disorders of communication (2nd ed.). London: Code and Whurr.

Pring, T., Hamilton, A., Harwood, A., \& Macbride, L. (1993). Generalization of naming after picture word matching tasks - only items appearing in therapy benefit. Aphasiology, 7(4), 383-394.

Robson, J., Marshall, J., Pring, T., \& Chiat, S. (1998). Phonological naming therapy in jargon aphasia: Positive but paradoxical effects. Journal of the International Neuropsychological Society, 4(6), 675-686.

Sbisa, S., D'Andrea, J., Semenza, C., \& Tabossi, P. (2001). Rehabilitating anomia: A case study. Brain and Language, 79(1), 46-47.

Spencer, K. A., Doyle, P. J., McNeil, M. R., Wambaugh, J. L., Park, G., \& Carroll, B. (2000). Examining the facilitative effects of rhyme in a patient with output lexicon damage. Aphasiology, 14(5/6), 567-584.

Squires, E. J., Hunkin, N. M., \& Parkin, A. J. (1997). Errorless learning of novel associations in amnesia. Neuropsychologia, 35(8), 1103-1111.

Tallal, P., Merzenich, M., Miller, S., \& Jenkins, W. (1998). Language learning impairments: Integrating basic science, technology, and remediation. Experimental Brain Research, 123, 210-219.

Terrace, H. S. (1963). Discrimination learning with and without "errors." Journal of the Experimental Analysis of Behavior, 6(1), 1-27.

Wilson, B. A., Baddeley, A., Evans, J., \& Shiel, A. (1994). Errorless learning in the rehabilitation of memory impaired people. Neuropsychological Rehabilitation, 4(3), 307-326. 
Wilson, B., \& Evans, J. (1996). Error-free learning in the rehabilitation of people with memory impairments. Journal of Head Trauma Rehabilitation, 11(2), 54-64.

Winter, J., \& Hunkin, N. M. (1999). Re-learning in Alzheimer's disease. International Journal of Geriatric Psychiatry, 14(11), 987-990.

Wykes, T., Reeder, C., Corner, J., Williams, C., \& Everitt, B. (1999). The effects of neurocognitive remediation on executive processing in patients with schizophrenia. Schizophrenia Bulletin, 25(2), 291-307.

Manuscript received December 2002

Revised manuscript received January 2003 
옹 